05.5

\title{
Сверхтонкие взаимодействия в узлах меди диэлектрических и сверхпроводящих металлооксидов меди
}

\author{
(ㄷ Е.И. Теруков ${ }^{1,2}$, А.В. Марченко ${ }^{3}$, А.А. Лужков ${ }^{3}$, П.П. Серегин ${ }^{3,9}$, К.Б. Шахович³ \\ ${ }^{1}$ Физико-технический институт им. А.Ф. Иоффре РАН, Санкт-Петербург, Россия \\ ${ }^{2}$ Санкт-Петербургский государственный электротехнический университет „ЛЭТИ“, Санкт-Петербург, Россия \\ ${ }^{3}$ Российский государственный педагогический университет им. А.И. Герцена, Санкт-Петербург, Россия \\ ๑ E-mail: ppseregin@mail.ru
}

Поступило в Редакцию 13 сентября 2019 г.

В окончательной редакции 30 июля 2020 г.

Принято к публикации 1 августа 2020г.

Методом мессбауэровской спектроскопии на изотопах ${ }^{61} \mathrm{Cu}\left({ }^{61} \mathrm{Ni}\right)$ показано, что для диэлектрических металлооксидов двухвалентной меди наблюдается комбинированное квадрупольное и зеемановское взаимодействие ядер ${ }^{61} \mathrm{Ni}$ с локальными полями в узлах меди, тогда как для сверхпроводящих металлооксидов обнаружено только квадрупольное взаимодействие ядер ${ }^{61} \mathrm{Ni}$. Для всех металлооксидов найден одинаковый по величине валентной вклад в тензор градиента электрического поля на ядрах ${ }^{61} \mathrm{Ni}^{2+}$ и ${ }^{63} \mathrm{Cu}^{2+}$.

Ключевые слова: высокотемпературные сверхпроводники, эмиссионная мессбауэровская спектроскопия, ЯМР, тензор градиента электрического поля.

DOI: 10.21883/PJTF.2020.21.50199.18037

Значительная часть высокотемпературных сверхпроводников (ВТСП) на основе металлооксидов меди была получена из антиферромагнитных соединений типа $\mathrm{YBa}_{2} \mathrm{Cu}_{3} \mathrm{O}_{6}$ [1], $\mathrm{La}_{2} \mathrm{CuO}_{4}$ [2], $\mathrm{Nd}_{2} \mathrm{CuO}_{4}$ [3], $\mathrm{Ca}_{2} \mathrm{CuO}_{2}$ [4], $\mathrm{SrCuO}_{2}$ [5], $\mathrm{Ca}_{2} \mathrm{CuO}_{2} \mathrm{Cl}_{2}$ [6] и $\mathrm{Sr}_{2} \mathrm{CuO}_{2} \mathrm{Cl}_{2}$ [7] путем гетеровалентного замещения (например, переход от $\mathrm{La}_{2} \mathrm{CuO}_{4}$ к $\left.\mathrm{La}_{2-x} \mathrm{Sr}_{x} \mathrm{CuO}_{4}\right)$ или изменения степени окисления атомов меди (например, переход от $\mathrm{YBa}_{2} \mathrm{Cu}_{3} \mathrm{O}_{6}$ к $\left.\mathrm{YBa}_{2} \mathrm{Cu}_{3} \mathrm{O}_{7-x}\right)$. Все эти соединения представляют интерес в плане изучения возможной связи между магнетизмом и сверхпроводимостью.

Мессбауэровская спектроскопия широко используется для изучения ВТСП на основе металлооксидов меди [8]. В частности, для исследования комбинированного магнитного и электрического квадрупольного взаимодействия в узлах меди решеток ВТСП целесообразно использовать эмиссионную мессбауэровскую спектроскопию на изотопах ${ }^{61} \mathrm{Cu}\left({ }^{61} \mathrm{Ni}\right)$, поскольку в этом случае зонд ${ }^{61} \mathrm{Ni}^{2+}$, образующийся после радиоактивного распада ${ }^{61} \mathrm{Cu}$, оказывается в узлах меди. В настоящей работе метод эмиссионной мессбауэровской спектроскопии на изотопах ${ }^{61} \mathrm{Cu}\left({ }^{61} \mathrm{Ni}\right)$ используется для исследования сверхпроводящих и диэлектрических соединений $\mathrm{Ca}_{1-x} \mathrm{Sr}_{x} \mathrm{CuO}_{2}, \mathrm{Ca}_{2} \mathrm{CuO}_{2} \mathrm{Cl}_{2}, \mathrm{SrCuO}_{2}, \mathrm{Sr}_{2} \mathrm{CuO}_{2} \mathrm{Cl}_{2}$, $\mathrm{YBa}_{2} \mathrm{Cu}_{3} \mathrm{O}_{7-x}, \mathrm{La}_{2-x} \mathrm{Sr}_{x} \mathrm{CuO}_{4}$ и $\mathrm{Nd}_{2-x} \mathrm{Ce}_{x} \mathrm{CuO}_{4}$.

Поликристаллические образцы готовились по методикам, описанным в [1-7]. Однофазность образцов контролировалась рентгенофазовым анализом. Критические температуры для сверхпроводящих образцов $\mathrm{YBa}_{2} \mathrm{Cu}_{3} \mathrm{O}_{6.9}, \mathrm{La}_{1.85} \mathrm{Sr}_{0.15} \mathrm{CuO}_{4}$ и $\mathrm{Nd}_{1.85} \mathrm{Ce}_{0.15} \mathrm{CuO}_{4}$ были равны 78, 37 и $22 \mathrm{~K}$ соответственно. Мессбауэровские источники готовились методом диффузии изотопа ${ }^{61} \mathrm{Cu}$ в готовую керамику при температурах $500-650^{\circ} \mathrm{C}$.
Мессбауэровские спектры ${ }^{61} \mathrm{Cu}\left({ }^{61} \mathrm{Ni}\right)$ регистрировались при $80 \mathrm{~K}$ на спектрометре SM 4201 TerLab. Стандартным поглотителем служил сплав $\mathrm{Ni}_{0.86} \mathrm{~V}_{0.14}$. Экспериментальные мессбауэровские спектры исследованных соединений представляют собой плохо разрешенные мультиплеты (рис. 1).

Для сверхпроводящих соединений следовало ожидать взаимодействия квадрупольного момента ядер ${ }^{61} \mathrm{Ni}$ с тензором градиента электрического поля (ГЭП) в узлах меди, и поэтому экспериментальные спектры представляют собой плохо разрешенные квадрупольные квинтеты. Поскольку диэлектрические соединения представляют собой антиферромагнетики с высокими значениями температуры Нееля ( 250-260 K для $\mathrm{La}_{2} \mathrm{CuO}_{4}$ [2,9,10], $\mathrm{Nd}_{2} \mathrm{CuO}_{4}$ [3,9,11], $\mathrm{SrCuO}_{2}$ [5], $\mathrm{Ca}_{2} \mathrm{CuO}_{2} \mathrm{Cl}_{2} \quad[6,9]$ и $\mathrm{Sr}_{2} \mathrm{CuO}_{2} \mathrm{Cl}_{2} \quad[7,9], \sim 418 \mathrm{~K}$ для $\mathrm{YBa}_{2} \mathrm{Cu}_{3} \mathrm{O}_{6}[1,12]$ и $\sim 540 \mathrm{~K}$ для $\left.\mathrm{Ca}_{2} \mathrm{CuO}_{2}[4,13]\right)$, тонкую структуру их мессбауэровских спектров в области температур ниже $200 \mathrm{~K}$ следует рассматривать как результат проявления эффектов комбинированного зеемановского и квадрупольного взаимодействия ядер ${ }^{61} \mathrm{Ni}$ c локальными полями.

В случае комбинированного магнитного и электрического взаимодействия собственные значения гамильтониана для каждого (основного и возбужденного) уровня ${ }^{61} \mathrm{Ni}$ могут быть найдены из соотношения

$$
\begin{aligned}
E_{m}^{I}= & m g H+\left[e Q U_{z z} / 4 I(2 I-1)\right] \\
& \times\left[3 m^{2}-I(I+1)\right]\left[\left(3 \cos ^{2} \theta-l\right) / 2\right] .
\end{aligned}
$$

Здесь $I-$ спин ядра, $Q-$ квадрупольный момент ядра, $H-$ магнитное поле на ядре, $U_{z z}-$ главная компонента тензора ГЭП на ядре, $\theta-$ угол между 


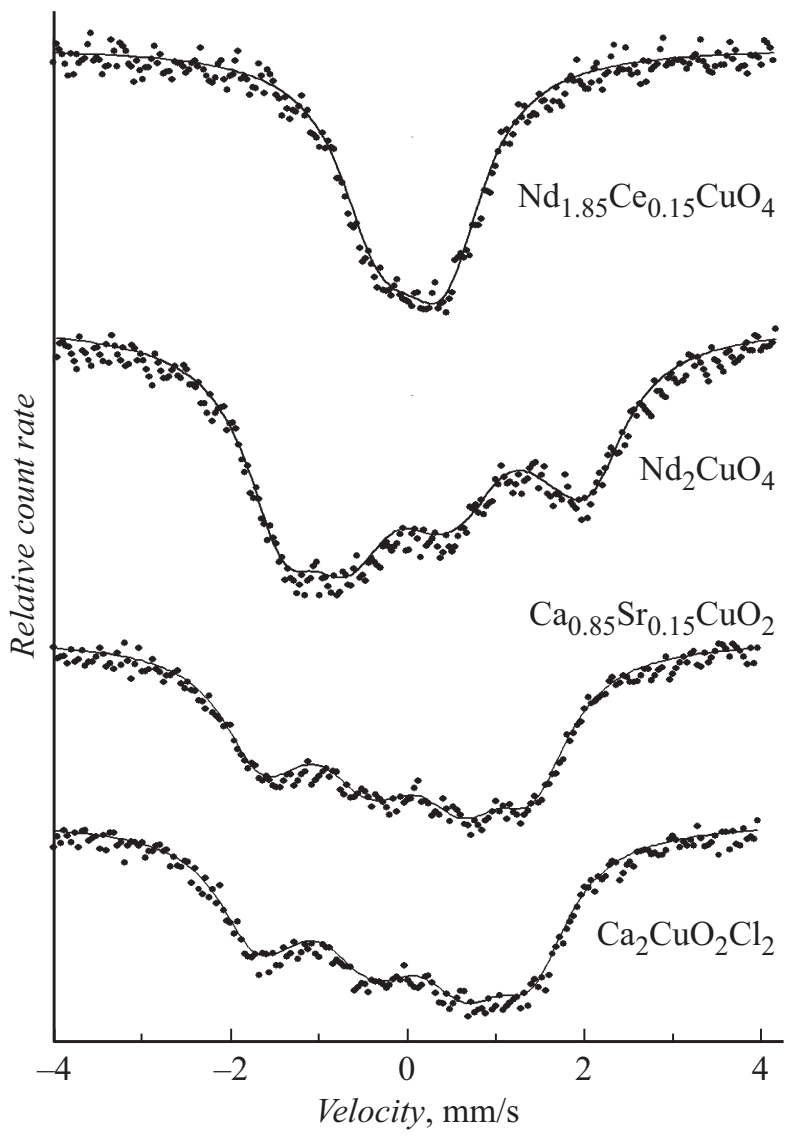

Рис. 1. Эмиссионные мессбауэровские спектры ${ }^{61} \mathrm{Cu}\left({ }^{61} \mathrm{Ni}\right)$ сверхпроводящей керамики $\mathrm{Nd}_{1.85} \mathrm{Ce}_{0.15} \mathrm{CuO}_{4}$ и диэлектрических керамик $\mathrm{Nd}_{2} \mathrm{CuO}_{4}, \mathrm{Ca}_{0.85} \mathrm{Sr}_{0.15} \mathrm{CuO}_{2}$ и $\mathrm{Ca}_{2} \mathrm{CuO}_{2} \mathrm{Cl}_{2}$. Сплошной линией показан расчетный спектр.

главной осью тензора ГЭП и направлением магнитного поля, $m-$ магнитное квантовое число, $g-$ гидромагнитное отношение (для ядра ${ }^{61} \mathrm{Ni}$ в основном состоянии $g=-0.070083 \mathrm{~mm} /(\mathrm{s} \cdot \mathrm{T})$, в возбужденном состоянии $\left.g_{e x}=0.0268 \mathrm{~mm} /(\mathrm{s} \cdot \mathrm{T})[8]\right)$. Расчетный спектр подгонялся к экспериментальному методом наименьших квадратов. Подгоночными параметрами были параметры гамильтониана $H$ и $U_{z z}\left[\left(3 \cos ^{2} \theta-1\right) / 2\right]$, общие для обоих ядерных уровней, а также интенсивности линий. При этом множитель $\left(3 \cos ^{2} \theta-1\right) / 2$ был принят нами равным единице, поскольку главный вклад в ГЭП вносят валентные $d$-электроны зондов и для них $z$-ось тензора направлена вдоль спина, который в свою очередь ориентирован вдоль магнитного поля. Согласие расчетного и экспериментального спектров оценивалось по критерию $\chi^{2}$.

В результате обработки мессбауэровских спектров были получены величины постоянной квадрупольного взаимодействия $C_{\mathrm{Ni}}=e Q^{61} U_{z z}$, напряженности магнитного поля $H$, угла $\theta$ между главной осью тензора ГЭП и направлением магнитного поля и параметра асимметрии тензора ГЭП $\eta=\left(U_{y y}-U_{x x}\right) / U_{z z}$, где $x, y$, $z$ - главные оси тензора ГЭП на ядре-зонде, $U_{x x}, U_{y y}$,
$U_{z z}$ - компоненты диагонализированного тензора ГЭП, $Q^{61}$ - квадрупольный момент ядра ${ }^{61} \mathrm{Ni}$ в основном состоянии. Для всех изученных соединений получено $\eta=0$, для всех антиферромагнитных соединений $\theta=0^{\circ}$ и величины $H$ находились в пределах от 8.5 до $10.0 \mathrm{~T}$. Полученные значения $C_{\mathrm{Ni}}$ представлены на рис. $2, a$.

Для центров ${ }^{63} \mathrm{Cu}^{2+}$ и замещающих их центров ${ }^{61} \mathrm{Ni}^{2+}$ тензор ГЭП на ядре-зонде создается ионами кристаллической решетки (тензор решеточного ГЭП) и несферической валентной оболочкой атома-зонда (тензор валентного ГЭП), причем, когда ориентация главных осей всех тензоров совпадает, имеем

$$
e Q U_{z z}=e Q(1-\gamma) V_{z z}+e Q(1-R) W_{z z},
$$

где $e Q-$ квадрупольные моменты ядер ${ }^{61} \mathrm{Ni}$ или ${ }^{63} \mathrm{Cu}$, $U_{z z}, V_{z z}, W_{z z}$ - главные компоненты тензоров суммарного, решеточного и валентного ГЭП для зонда, а $\gamma$ и $R$ - коэффициенты Штернхеймера для зонда.

Расчет параметров тензора решеточного ГЭП проводился в рамках модели точечных зарядов, а параметры элементарной ячейки для исследованных соединений были взяты из работ [14-17]. Для диэлектрических оксидов заряды атомов считались равными их традиционной валентности, а для сверхпроводящих оксидов выбор моделей основывался на данных [13]. Для всех соединений тензоры решеточных ГЭП в узлах меди оказались диагональными в кристаллографических осях,

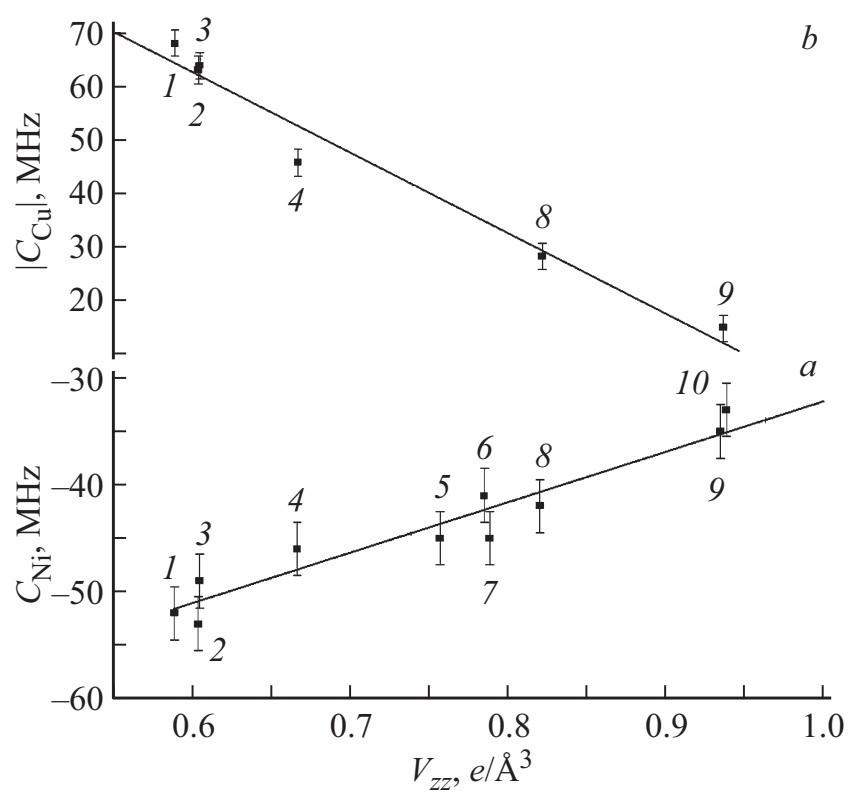

Рис. 2. Зависимости постоянной квадрупольного взаимодействия для центров ${ }^{61} \mathrm{Ni}$ в узлах меди $C_{\mathrm{Ni}}(a)$ и для центров ${ }^{63} \mathrm{Cu}$ в узлах меди $\left|C_{\mathrm{Cu}}\right|(b)$ от главной компоненты $V_{z z}$ тензора решеточного ГЭП в этих узлах. Данные для $\left|C_{\mathrm{Cu}}\right|$ взяты из работ [18-20]. Обозначения узлов меди в решетках: $1-\mathrm{La}_{1.85} \mathrm{Sr}_{0.15} \mathrm{CuO}_{4}, 2-\mathrm{Cu}(2)$ в $\mathrm{YBa}_{2} \mathrm{Cu}_{3} \mathrm{O}_{6.9}, 3-\mathrm{La}_{2} \mathrm{CuO}_{4}$, $4-\mathrm{Cu}(2)$ в $\mathrm{YBa}_{2} \mathrm{Cu}_{3} \mathrm{O}_{6}, 5-\mathrm{Sr}_{2} \mathrm{CuO}_{2} \mathrm{Cl}_{2}, 6-\mathrm{Ca}_{2} \mathrm{CuO}_{2} \mathrm{Cl}_{2}$, $7-\mathrm{Nd}_{1.85} \mathrm{Ce}_{0.15} \mathrm{CuO}_{4}, 8-\mathrm{Nd}_{2} \mathrm{CuO}_{4}, 9-\mathrm{SrCuO}_{2}, 10-$ $\mathrm{Ca}_{0.85} \mathrm{Sr}_{0.15} \mathrm{CuO}_{2}$. 
$\eta=0$ и главные оси тензоров направлены по кристаллографической оси $c$.

На рис. 2, $a$ приведена зависимость $C_{\mathrm{Ni}}$ для центров ${ }^{61} \mathrm{Ni}^{2+}$ в узлах меди от $V_{z z}$ в этих узлах для всех исследованных соединений. Зависимость на рис. 2,a линейна и определяется соотношением

$$
C_{\mathrm{Ni}}=49 V_{z z}-81
$$

(здесь и далее величины $C_{\mathrm{Ni}}$ приводятся в $\mathrm{MHz}$, a $V_{z z}-$ в $\left.e / \AA^{3}\right)$.

Из соотношения (2) следует, что линейная зависимость (3) является следствием одинаковой величины валентной составляющей в ГЭП для зонда $\mathrm{Ni}^{2+}$ в узлах двухвалентной меди $e Q^{61}(1-R) W_{z z}=-81(2) \mathrm{MHz}$. Сoотношение (3) свидетельствует также о противоположных знаках валентного и решеточного вкладов в формуле (2) для центров $\mathrm{Ni}^{2+}$ и выполнении соотношения $\left|(1-R) W_{z z}\right|>\left|(1-\gamma) V_{z z}\right|$ для этих центров.

Для сравнения на рис. 2, $b$ приведена зависимость абсолютной величины постоянной квадрупольного взаимодействия для центров ${ }^{63} \mathrm{Cu}^{2+}\left(C_{\mathrm{Cu}}=e Q^{63} U_{z z}\right.$, где $\mathrm{Q}^{63}-$ квадрупольный момент ядра $\left.{ }^{63} \mathrm{Cu}\right)$ в узлах меди от значения $V_{z z}$ в этих узлах. При построении этой зависимости использовались данные ядерного магнитного резонанса на изотопе ${ }^{63} \mathrm{Cu}[18-20]$. Видно, что зависимость на рис. $2, b$ линейна и определяется соотношением

$$
\left|C_{\mathrm{Cu}}\right|=-150 V_{z z}+153 \text {. }
$$

Из соотношения (2) следует, что линейная зависимость (4) является следствием одинаковой величины валентной составляющей в тензоре ГЭП для зонда $\mathrm{Cu}^{2+}$ в металлооксидах двухвалентной меди $e Q^{63}(1-R) W_{z z}=153(2) \mathrm{MHz}$. Соотношение (4) также свидетельствует о противоположных знаках валентного и решеточного вкладов в формуле (2) для центров $\mathrm{Cu}^{2+}$ и выполнении соотношения $\left|(1-R) W_{z z}\right|>\left|(1-\gamma) V_{z z}\right|$ для этих центров.

Таким образом, для диэлектрических металлооксидов двухвалентной меди обнаружено комбинированное электрическое и магнитное взаимодействие ядер ${ }^{61} \mathrm{Ni}$ с локальными полями в узлах меди, тогда как для сверхпроводящих металлооксидов спектры соответствуют взаимодействию квадрупольного момента ядер ${ }^{61} \mathrm{Ni}$ с тензором ГЭП. Для всех металлооксидов меди наблюдаются линейные зависимости постоянных квадрупольного взаимодействия на ядрах ${ }^{61} \mathrm{Ni}$ и ${ }^{63} \mathrm{Cu}$ от расчетных значений главной компоненты тензора решеточного ГЭП в узлах меди. Этот факт объясняется тем, что для зондов ${ }^{61} \mathrm{Ni}^{2+}$ и ${ }^{61} \mathrm{Cu}^{2+}$ величина вклада валентной составляющей в суммарный тензор ГЭП не зависит от состава металлооксида.

\section{Конфликт интересов}

Авторы заявляют, что у них нет конфликта интересов.

\section{Список литературы}

[1] Khan M.Z., Malmivirta M., Zhao Y., Wu X., Jha R., Awana V.P.S., Huhtinen H., Paturi P. // Physica C. 2018. V. 555. P. $15-23$.

[2] Sukumar M., Kennedy L., Vijaya J., Al-Najar B., Bououdina M. // Ceram. Int. 2018. V. 44. P. 18113.

[3] Horio M., Krockenberger Y., Yamamoto K., Yokoyama Y., Takubo K., Hirata Y., Sakamoto S., Koshiishi K., Yasui A. // Phys. Rev. Lett. 2018. V. 120. P. 257001.

[4] Di Castro D., Ridolfi F., Aruta C., Tebano A., Yang N., Balestrino G. // Phys. Rev. Lett. 2015. V. 115. P. 147001.

[5] Bounoua D., Saint-Martin R., Petit S., Bourdarot F. // Physica B. 2018. V. 536. P. 323-326.

[6] Lebert B.W., Dean M.P.M., Nicolaou A., Pelliciari J., Dantz M., Schmitt T., Yu R. // Phys. Rev. B. 2017. V. 95. P. 155110.

[7] Petersen J.C., Farahani A., Sahota D.G., Liang R., Dodge J.S. // Phys. Rev. B. 2017. V. 96. P. 115133

[8] Seregin N., Marchenko A., Seregin P. Emission Mössbauer spectroscopy. Electron defects and Bose-condensation in crystal lattices of high-temperature supercomductors. Saarbrücken: LAP LAMBERT, 2015. 325 p.

[9] Hechel D., Felner I. // Physica C. 1994. V. 235-240. P. 16011602.

[10] Keimer B., Aharony A., Auerbach A., Biegeneau R.J., Cassanho A., Endoh Y., Erwin R.W., Kastner M.A., Shirane G. // Phys. Rev. B. 1992. V. 45. P. 7430-7435.

[11] Matsuda M., Yamada K., Kakarai K., Kadowaki H., Thurston T.R., Endoh Y., Hidaka Y., Birgeneau R.J., Kastner M.A., G'ehring P., Moudden A.H., Shirane G. // Phys. Rev. B. 1990. V. 42. P. 10098-10107.

[12] Petigrand D., Collin G. // Physica C. 1988. V. 153-155. P. 192-193.

[13] Vaknin D., Caignol E., Davies P.K., Fischer J.E., Johnston D.C., Goshorn D.P. // Phys. Rev. B. 1989. V. 39. P. 9122-9125.

[14] Siegrist T., Zahurak S.M., Murphy D.W., Roth R.S. // Nature. 1988. V. 334. P. 231-232.

[15] Yvon K., Francois M. // Z. Phys. D. 1989. V. 76. P. 413-444.

[16] Zhou X., Wu F., Yin D., Liu W., Dong C., Li J., Zhu W., Jia S., Yao Y., Zhao Z. // Physica C. 1994. V. 233. P. 311-320.

[17] Haas H., Correia J.G. // Hyperfine Interact. 2007. V. 176. P. 9-13.

[18] Takatsuka T., Kumagai K., Nakajima H., Yamanaka A. // Physica C. 1991. V. 185-189. P. 1071-1072.

[19] Yoshinari Y., Yasuoka H., Shimizu T., Takagi H., Tokura Y., Uchida S. // J. Phys. Soc. Jpn. 1990. V. 59. P. 36-39.

[20] Shimizu T. // J. Phys. Soc. Jpn. 1993. V. 62. P. 772-778. 